\title{
El losartan reduce la morbimortalidad de hipertensos con hipertrofia ventricular izquierda con respecto al atenolol
}

Cardiovascular morbidity and mortality in the Losartan Intervention For Endpoint reduction in hypertension study (LIFE):a randomized trial against atenolol. Dahlof B; Devereux RB;Kjeldsen SE;Julius S; et al. Lancet 2002; 359:995-1003

\section{Objetivo}

Evaluar si el antagonista de los receptores de angiotensina II (ARA) Losartan es más efectivo que el beta-bloqueantes (BB) Atenolol, disminuyendo la morbimortalidad cardiovascular en pacientes con hipertensión arterial (HTA) e Hipertrofia Ventricular Izquierda (HVI).

Diseño

Ensayo clínico aleatorizado, doble ciego, de grupos paralelos comparativo entre dos drogas.

Lugar

Estudio multicéntrico realizado en Dinamarca, Finlandia, Noruega, Suecia y EE.UU.

Pacientes

Participaron 9193 pacientes de 55-80 años con HTA esencial (TA sentado $160-200 / 95-115 \mathrm{mmHg}$ ) e HVI determinada por electrocardiograma (ECG).Se excluyeron pacientes con eventos vasculares en los 6 meses previos, con angina de pecho que requiera BB o bloqueantes cálcicos, insuficiencia cardíaca o fracción de eyección $\leq 40 \%$, o con indicación de ARA, inhibidores de la enzima convertidora de angiotensina (IECA), BB o hidroclorotiazida (HCTZ).

Intervención

Tras 1-2 semanas de placebo se aleatorizó a los pacientes a recibir losar$\tan (50 \mathrm{mg} / \mathrm{día}$ ) o atenolol (50mg/día) por al menos 4 años y hasta que 1040 pacientes tuvieran un evento cardiovascular primario.Con cualquier tratamiento, si laTA era $\geq 140 / 90$ el 1er paso fue asociar $12,5 \mathrm{mg} / \mathrm{día}$ de HCTZ, el 2do fue duplicar las droga en estudio y el 3ro duplicar la HCTZ y/o asociar una droga diferente a IECA o las drogas en estudio. Medición de los resultados principales

Resultado primario combinado, contabilizando el primero de los siguientes eventos: muerte cardiovascular, infarto agudo de miocardio (IAM) o accidente cerebrovascular (ACV). Se realizó un análisis multivariable* ajustado por el grado de HVI y el puntaje de riesgo de Fram- ingham. ${ }^{1}$ Los resultados fueron evaluados por dos revisores ciegos a la intervención y los desacuerdos resueltos por consenso.

Resultados Principales

De los 9222 pacientes aleatorizados un $2 \%$ por grupo salieron del estudio pero igualmente se analizaron por intención de tratar.Las características basales de los pacientes fueron muy similares.Tras 4,8 años de seguimiento medio el 84 y $80 \%$ continuaron recibiendo losartan y atenolol respectivamente. La distribución de drogas adicionales no varió significativamente entre grupos. Con losartan la TA bajó en promedio $30,2 / 16,6 \mathrm{mmHg}$ y con atenolol 29,1/16,8 $\mathrm{mmHg}$. La diferencia de 1 $\mathrm{mmHg}$ en la TA sistólica a favor del losartan no modificó los resultados analizados.Los resultados pueden verse en la Tabla.

El análisis de las curvas de sobrevida muestra un temprano y progresivo aumento de las diferencias en el tiempo para el resultado primario y más aún para ACV. La diferencia es menor en mortalidad cardiovascular e inexistente para IAM. También fueron menores las internaciones y los procedimientos de revascularización. En el subgrupo de pacientes de menor riesgo (sin enfermedad vascular ni diabetes) la eficacia fue similar (NNT 61, IC 95\% 33-401).La regresión de la HVI por ECG (utilizando los criterios de Sok olow-Lyon) fue del $14 \%$ con Losartan y de $10 \%$ con Atenolol $(p<0,0001)$. El análisis mostró que menos de un tercio del efecto sobre el resultado primario se debió a la regresión de la HVI.La suspensión del tratamiento fue significativamente menos frecuente con losartan, tanto la debida a cualquier efecto adverso (EA) como los relacionados a la drogas. En la tabla se mencionan los EA preespecificados estadísticamente significativos, aunque también resultaron significativamente menos frecuentes con losartan los siguientes EA de frecuencia $>5 \%$ :albuminuria, hiperglucemia, astenia, disnea, edema distal y neumonía. Conclusión

El losartan previno más la morbimortalidad cardiovascular que el atenolol para una reducción similar de la TA y fue mejor tolerado.El losartan parece conferir beneficios más allá de la reducción de la TA.

\begin{tabular}{|c|c|c|c|c|c|c|c|}
\hline \multirow[t]{2}{*}{ Resultados } & \multicolumn{2}{|c|}{ Losartan ( $n=586)$} & \multicolumn{2}{|c|}{ Atenolol $(n=609)$} & \multirow[t]{2}{*}{ HR $^{* *}$ ajustado (IC95\%) } & \multirow[t]{2}{*}{ p } & \multirow[t]{2}{*}{ 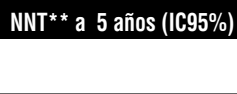 } \\
\hline & $\begin{array}{c}n(\%) \\
\text { (en } 5 \text { años) }\end{array}$ & $\begin{array}{c}\% \text { pacientes } \\
\text { año }\end{array}$ & $\begin{array}{c}n(\%) \\
\text { (en } 5 \text { años) }\end{array}$ & $\begin{array}{c}\% \text { pacientes } \\
\text { año }\end{array}$ & & & \\
\hline 1ario combinado & $508(11 \%)$ & $23,8 \%$ & $588(13 \%)$ & $27,9 \%$ & $0,87(0,77-0,98)$ & 0,021 & $56(32-217)$ \\
\hline - Muerte CV & $204(4 \%)$ & $9,2 \%$ & $234(5 \%)$ & $10,6 \%$ & $0,89(0,73-1,07)$ & 0,206 & -- \\
\hline - ACV & $232(5 \%)$ & $10,8 \%$ & $309(7 \%)$ & $14,5 \%$ & $0,75(0,63-0,89)$ & 0,001 & $59(38-136)$ \\
\hline - IAM & $198(4 \%)$ & $9,2 \%$ & $188(4 \%)$ & $8,7 \%$ & $1,07(0,88-1,39)$ & 0,491 & --- \\
\hline Mortalidad total & $383(8 \%)$ & $17,3 \%$ & $431(9 \%)$ & $19,6 \%$ & $0,90(0,78-1,03)$ & 0,128 & --- \\
\hline Nuevos diabéticos\& & $241(6 \%)$ & $13,0 \%$ & $319(8 \%)$ & $17,4 \%$ & $0,75(0,63-0,88)$ & 0,001 & $58(37-135)$ \\
\hline EA $\quad$ Bradicardia & $66(1 \%)$ & $<$ & $391(9 \%)$ & $>$ & & $<0,001$ & $14(13-16)$ \\
\hline Frialdad distal & $178(4 \%)$ & $<$ & $269(6 \%)$ & $>$ & & $<0,001$ & $50(35-89)$ \\
\hline Hipotensión & $121(3 \%)$ & $>$ & $75(2 \%)$ & $<$ & & 0,001 & $100(63-248)$ \\
\hline Disfunción sexual & $164(4 \%)$ & $<$ & $214(5 \%)$ & $>$ & & 0,009 & $91(52-343)$ \\
\hline
\end{tabular}

CV: cardiovascular, EA:Efectos Adversos preespecificados en el análisis y estadísticamente significativos, ${ }^{\star *}$ NNT: $N^{\circ}$ necesario para tratar para evitar 1 evento (dato calculado por el comentador mediante Stata 6.0 A partir de los valores absolutos sin redondeos), HR, del inglés hazard ratio (similar al riesgo relativo en los análisis de sobrevida); > ó < :mayor ó menor frecuencia comparativa, \#Revascularización miocárdica o periférica, \& Nuevos casos de diabetes (definidos por criterios de la OMS de 1985).

Fuente de financiamiento:Laboratorio Merck.

\section{Comentario}

Los hipertensos tratados tienen menos complicaciones cardiovasculares que los no tratados, pero más que pacientes similares normotensos. Es to podría deberse al fracaso para normalizar la TA y/o al daño residual de órganos.Sin embargo los resultados se mantienen si se ajusta por riesgo cardiovascular, $\mathrm{HVI}$ y aún por la mínima diferencia de TA. Hasta el momento ningún tratamiento de la HTA había prevenido la morbimortalidad cardiovascular más allá que las reducciones de TA obtenidas con diuréticos y beta bloqueantes,2,3 y el reporte del VI JNC4 estableció en 1997 que no había ventajas con los nuevos agentes pues las tiazidas y BB habían probado disminuir la morbimortalidad frente al placebo. En este estudio, el losartan demostró reducir aún más la morbimortalidad cardiovascular (un 13\% en términos relativos) que un antihipertensivo establecido de reconocidas propiedades cardioprotectoras como el atenolol. En el estudio STOP los BB asociados a diuréticos redujeron un $40 \%$ la morbimortalidad cardiovascular frente al placebo. 5

Además, un dato no menor para los pacientes es la mejor tolerancia de losartan, ocasionando significativamente menor abandono que el atenolol y menos EA como disfunción sexual, frialdad distal e hipotensión.

El reporte separado de los pacientes diabéticos mostró beneficios aún más marcados: $24 \%$ de reducción del resultado primario, $39 \%$ de la mortalidad total, $37 \%$ la mortalidad cardiovascular y $40 \%$ de la internación por insuficiencia cardíaca.Las diferencias fueron aún mayores en el subgrupo de pacientes diabéticos virgen de tratamiento antihipertensivo al inicio. Dado que estos impactantes resultados podrían modificar la prác- tica es necesario considerar plausibilidad biológica, la validez interna* externa* de los mismos. La angiotensina II produce daños que van más allá de los daños por HTA6 y antagonizarla no sólo mejora la TA sino también la evolución de la insuficiencia cardíaca y la perfusión de órganos vitales. El estudio LIFE abona fuertemente el concepto que bloquear el sistema renina-angiotensina puede proveer protección más allá del control de la TA, que en este estudio fue similar en ambos grupos y el ajuste por las nimias diferencias halladas no alteró los resultados. Por otro lado, la mayor regresión de la HVI en el grupo losartan (también observada en otros estudios) sólo explicaría una parte de ese efecto.La microalbuminuria, que precede a la nefropatía diabética manifiesta, también fue significativamente reducida por el losartan. A diferencia del estudio HOPE 7 en el LIFE los grupos estuvieron balanceados o incluso hubo un riesgo ligeramente mayor en el grupo losartan.La excepción fue la fibrilación auricular que la tuvieron 24 pacientes menos que en grupo atenolol. Aún así la prevención de 77 ACV fue altamente significativa con losartan y excede en mucho este desequilibrio. Es llamativa la menor incidencia de diabetes en el grupo losartan.Dado que no existe razón para que el bloqueo de los receptores de angiotensina alteren la mayor insulino-resistencia de la HTA esencial, podría especularse que los BB, que reducen la insulino-sensibilidad, 8 fueran los responsables de este fenómeno. Estos resultados se obtuvieron aún cuando menos de la mitad de los pacientes recibieron $<100 \mathrm{mg} /$ día de las drogas en estudio. Considerando que en ciertas circunstancias los ARA proveen mejor protección a dosis 


\section{El losartan reduce la morbimortalidad de hipertensos diabéticos con hipertrofia ventricular izquierda respecto al atenolol}

Cardiovascular morbidity and mortality in patients with diabetes in the Losartan Intervention For Endpoint reduction in hypertension study (LIFE):a randomized trial against atenolol. Lindholm LH;Ibsen H;Dahlof B;Devereux RB;et al. Lancet 2002;359:1004-10..

\section{Objetivo}

Evaluar si el losartan es más efectivo que el atenolol, disminuyendo la morbimortalidad cardiovascular en pacientes diabéticos con hipertensión arterial $(\mathrm{HTA})$ e hipertrofia de ventrículo izquierdo (HVI).

\section{Diseño}

Subgrupo preespecificado de pacientes diabéticos del estudio LIFE (ver página previa).

Lugar

Estudio multicéntrico realizado en Dinamarca, Finlandia, Noruega, Suecia y EE.UU.

\section{Pacientes}

Participaron 1195 pacientes diabéticos (13\% de la cohorte del LIFE) con medias de edad de $67 \pm 7$ años, de TA prealeatorización de $177 / 96 \mathrm{mmHg}$ y de seguimiento de 4,7 años.Todos con HTA e HVI por ECG.

\section{Intervención}

Ver intervención de LIFE (página previa).

\section{Medición de los resultados principales}

Resultado primario combinado (muerte cardiovascular, infarto de miocardio [IAM] o accidente cerebrovascular [ACV]) ajustado por el grado de HVI y el puntaje de Framingham. El análisis fue por intención de tratar. Resultados Principales

Las características basales de los pacientes y sus tratamientos para la diabetes y dislipidemia fueron muy similares entre los grupos. El índice de masa corporal, el puntaje de Framingham y la prevalencia de enfermedad cardiovascular fueron mayores que la población del LIFE sin diabetes.En el grupo losartan la TA bajó a 146/79 (reducción 31/17) mmHg y en el grupo atenolol bajó a 148/79 (reducción 28/17) mmHg. La diferencia de $2 \mathrm{mmHg}$ en la TA sistólica a favor del losartan tras el ajuste no modificó los resultados analizados.Los resultados pueden verse en la Tabla. También fueron menores las internaciones y los procedimientos de revascularización. La regresión de la HVI por ECG (utilizando el criterio de Sokolow-Lyon) fue del $13,6 \%$ con losartan y de $5,6 \%$ con atenolol $(p<0,0001)$.La suspensión fue menos frecuente con losartan $(27 \%)$ que con atenolol (32\%) pero no alcanzó significancia estadística.De los eventos adversos (EA) preespecificados sólo la bradicardia fue estadísticamente significativa. De los siguientes EA de frecuencia $>5 \%$ resultaron significativamente menos frecuentes con losartan la albuminuria, la hipokalemia y el vértigo;y menos frecuente con atenolol la angina de pecho.

Conclusión

En pacientes diabéticos con HTA e HVI, el losartan redujo más la morbimortalidad cardiovascular y la mortalidad por todas las causas que el atenolol. El losartan parece conferir beneficios más allá de la reducción de la TA.

\begin{tabular}{|c|c|c|c|c|c|c|c|}
\hline \multirow[t]{2}{*}{ Resultados } & \multicolumn{2}{|c|}{ Losartan $(n=586)$} & \multicolumn{2}{|c|}{ Atenolol $(n=609$ ) } & $\mathrm{HR}^{* *}$ ajustado (IC95\%) & $\mathbf{p}$ & NNT** a 5 años (IC95\%) \\
\hline & $\begin{array}{c}n(\%) \\
\text { (en } 5 \text { años) }\end{array}$ & $\begin{array}{l}\% \text { pacientes } \\
\text { año }\end{array}$ & $\begin{array}{c}n(\%) \\
\text { (en } 5 \text { años) }\end{array}$ & $\begin{array}{l}\% \text { pacientes } \\
\text { año }\end{array}$ & & & \\
\hline 1ario combinado & $103(18 \%)$ & $39,2 \%$ & $139(23 \%)$ & $53,6 \%$ & $0,76(0,58-0,98)$ & 0,031 & $19(10-141)$ \\
\hline - Muerte CV & $38(6 \%)$ & $13,6 \%$ & $61(10 \%)$ & $21,8 \%$ & $0,63(0,42-0,95)$ & 0,028 & $23(15-236)$ \\
\hline - ACV & $51(9 \%)$ & $19,0 \%$ & $65(11 \%)$ & $24,5 \%$ & $0,79(0,55-1,14)$ & 0,204 & --- \\
\hline - IAM & $41(7 \%)$ & $15,2 \%$ & $50(8 \%)$ & $18,7 \%$ & $0,83(0,55-1,25)$ & 0,373 & --- \\
\hline Mortalidad total & $63(11 \%)$ & $22,5 \%$ & $104(17 \%)$ & $37,2 \%$ & $0,61(0,45-0,84)$ & 0,002 & $16(10-41)$ \\
\hline
\end{tabular}

$\mathrm{CV}$ : cardiovascular, **NNT: ${ }^{\circ}$ necesario para tratar para evitar 1 evento (dato calculado por el comentador mediante Stata 6.0 A partir de los valores absolutos sin redondeos), HR, del inglés hazard ratio (similar al riesgo relativo en los análisis de sobrevida)

máximas ${ }^{9}$ es válido preguntarse si una mayor dosis hubiera mejorado el beneficio del losartan, ya que la incidencia de eventos primarios sigue siendo alta aún en este grupo, y podría aspirar a beneficios adicionales. Esta consideración es aún más atinada en el caso de los diabéticos pero menos del $40 \%$ alcanzaron una TA sistólica $<140 \mathrm{mmHg}$.Teniendo en cuenta las recomendaciones actuales de bajarla a menos de $130 \mathrm{mmHg}$ y debido a su particular perfil de riesgo, parece justificado un tratamiento más agresivo.Con respecto a la validez externa* debe mencionarse que en el LIFE, como ocurre en la práctica, la monoterapia fue generalmente insuficiente, y la adición de HCTZ al losartan parece no aumentar los efectos adversos.Debe destacarse que la población estudiada, además de ser fundamentalmente de raza blanca, se compone de hipertensos con HVI.Esta situación es frecuente especialmente en diabéticos $(>50 \%$ la padece $)^{10}$ debido al estímulo independiente de la enfermedad sobre la hipertrofia. Es interesante destacar que, incluso en el subgrupo de pacientes de menor riesgo del LIFE, la magnitud del beneficio fue consistente, estadística y clínicamente significativa.Considerando la similar fisiopatología de los eventos en todos los pacientes y que la regresión de la HVI no explica todo el beneficio parece correcto extrapolar los beneficios a hipertensos de menor riesgo. En estos pacientes de menor riesgo de eventos lo que disminuye es el beneficio absoluto, con el consiguiente aumento del NNT (por su menor frecuencia de eventos que en pacientes de riesgo, voy a necesitar tratar a muchos más para obtener el mismo beneficio).Por el contrario, entre los diabéticos el impacto es enorme a juzgar por los bajos NNT.Se necesitó tratar a 16 diabéticos por casi cinco años para prevenir una muerte en relación al atenolol.También parece apropiado extrapolar los resultados del losartan a otros ARA.Si bien las propiedades uricosúricas del losartan observadas en el LIFE pueden ser una ventaja comparativa en algunos pacientes, es improbable que expliquen todos los beneficios. En el estudio HOPE el IECA ramipril redujo $22 \%$ los eventos cardiovasculares en pacientes con diabetes y un factor de riesgo cardiovascular adicional.La evidencia actual sugiere que el efecto beneficioso de los IECA puede al menos ser reproducido por los ARA. El losartan e irbesartan demostraron ser renoprotectores en pacientes con diabetes y nefropatía independientemente de su efecto sobre la TA. ${ }^{11-13}$ En el estudio MICRO-HOPE14 el ramipril redujo $24 \%$ la nefropatía manifiesta en diabéticos. Aunque el irbesartan parecería superior (la redujo un 66\%) no existen estudios que comparen ARA con IECA.En el estudio CALM 15 el candesartan combinado con lisinopril por 24 semanas redujo más la TA y la albumina/creatinina que cada droga por separado. A la luz de la evidencia expuesta en pacientes hipertensos con HVI y en particular los diabéticos, los ARA o los IECA deberían ser considerados agentes de primera línea dada su probada eficacia previniendo morbimortalidad cardiovascular y la progresión de la nefropatía, poniendo atención no sólo en el control de la TA sino en la corrección otros factores de riesgo cardiovascular. La aplicación de esta conclusión a grupos de menor riesgo debiera ser comprobada.El bloqueo dual del sistema renina-angiotensina con un ARA y un IECA parece prometedor. También merece futuras investigaciones la comparación de los efectos de los IECA con los ARA.

\section{Dr. Agustín Ciapponi [ Unidad de Medicina Familiar y Preventiva.Hospital Italiano de Buenos Aires ]}

\section{Referencias}

1. Andersson KM, Wilson PWF, Odell PM, KanneWB.An updated coronary risk profile:a statement for health proessionals.Circulation 1991;83:356-62.

2. Neal B, MacMahon S, Chapman N.Effects of ACE inhibitors, calcium antagonists, and other tbod-pressure-lowering drugs: results of prospectively designed owerviews of randomised trials.Blood Pressure Lowering Treatment Tiralists' Collaboration. Lancet 2000;356:1955-64.

3. Hansson L, Lindholm LH, Ekbom T, et al.Randomised trial of old and new antibpertensive drugs in elderly patients:cardiovascular mortality and morbidity the Swedish Trial in Old Patients with Hypertension-2 study.Lancet 199æ554: $1751-56$.

4. The sixth report of the Joint National Committee on prevention detection, evaluation and treatment of high blood pressure.Arch Intern Med 199757:2413-46.

5. Dahlof B, Lindholm LH, Hansson I, Scherst6n B, Ekbom T, Wester P-0, Morbidity and mortality in the Swedish Trial in Old Patients with Hypertension (\$IP-Hypertension). Lancet 1991;338:1281-85.

6.Brunner HR, Laragh JH, Baer L, et al.Essential lypertension:renin and aldosterone, heart attack and stroke.NEJM 1972286:441-49.

7.The Heart Outcomes Prevention Evaluation Study Inestigators. Effects of an angiotensin-converting enzyme inhibitor, amipril, on cardiovascular events in high-risk patients.N Engl J Med2000;342:145-53. 8.Gress TW, Nieto FJ, Shahar E, et al.Hypertension and antihpertensive therapy as risk factors for type 2 diabetes mellitus.Atherosclerosis Risk in Communities Study.N Engl J Med.2000;342(13):905-12. 9.Parving HH, Lehnert H, Brochner-Mortensen J, et al. The effect of irbesartan on the development of diabetic nephropathy in patients with type 2 diabetes. N Engl J Mez001;345(12):870-8.

10.Bella IN, Devereux RB, Roman MJ, et al.Separate and joint effects of hypertension and diabetes mellitus on left entricular structure and function:the Strong Heart Study.Am J Cardiol 200187:1260-65. 1.Lewis EJ, Hunsicker LG, Clarke WR, et al.Renoprotective effect of the angiotensin-receptor antagonist irbesartan in patients with nephropathy due to type 2 diabetes.N Engl J Me\&001;345(12):851-60. 2.Brenner BM, Cooper ME, de Zeeuw D, et al:RENAAL Study Inestigators. Effects of losartan on renal and cardiovascular outcomes in patients with type 2 diabetes and nephropathy.N Engl J Med 2001 Sep 20;345(12):861-9

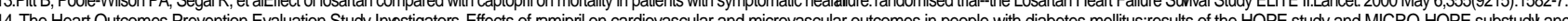
14. The Heart Outcomes Prevention Evaluation Study Inrestigators. Effects of ramipril on cardiovascular and microvascular outcomes in people with diabetes mellitus:results of the HOPE study and MICRO-HOPE substudk-ancet.
200;355:253-59. 15. Mogensen CE, Neldam S, Tikkanen I, et al.Randomised controlled trial of dual tockade of renin-angiotensin system in patients with lypertension, microalluminuria, and non-insulin dependent diabetes:the candesartan and
lisinopril microalbuminuria (CALM) study.BM. .2000:321:440-4. 